\title{
Trigger Tool na Segurança do Doente: Uma Revisão Sistemática de Literatura
}

\author{
Ludmila Pierdevara $^{a}$ Inês Margarida Ventura ${ }^{a}$ Margarida Eiras ${ }^{b}$ \\ Amélia Maria Brito Gracias ${ }^{c}$ \\ a Unidade Hospitalar de Lagos, Centro Hospitalar Algarve (CHA), Lagos, Portugal; ${ }^{b}$ Escola Superior de Tecnologia \\ em Saúde Lisboa (ESTeSL), Instituto Politécnico de Lisboa (IPL), Lisbon, Portugal; ' Unidade Hospitalar de Portimão, \\ Centro Hospitalar Algarve (CHA), Portimão, Portugal
}

\section{Palavras Chave \\ Global Trigger Tool · Segurança do paciente · Eventos adversos · Gestão de segurança · Dano do doente · Erros médicos}

\section{Resumo}

Introdução: A elevada incidência de eventos adversos (EA) é uma das situações preocupantes descritas na literatura sendo relacionada diretamente com o aumento dos custos com a prestação de cuidados de saúde e com o aumento do número de dias de internamento. A metodologia Global Trigger Tool (GTT) é relativamente nova mas tem-se mostrado promissora, em contextos internacionais, em ações de rastreio dos EA. Objetivo: Obter um corpo de evidência, reunindo um conjunto de informações acerca da utilização desta metodologia em contexto hospitalar. Metodologia: Tratase de uma revisão sistemática da literatura, baseada em estudos primários relacionados com esta temática, publicados entre o ano 2009 e 2014. Resultados: O estudo incluiu 8 estudos primários e os resultados evidenciam dados relevantes acerca da utilidade da ferramenta GTT no âmbito hospitalar. Conclusão: A evidência científica mostra que a aplicação do método GTT, como estratégia de monitorização dos EA, é uma ferramenta viável, uma vez que permite acompanhar a implementação de mudanças orientadas para a redução da ocorrência dos EA.

(c) 2017 The Author(s). Published by S. Karger AG, Basel on behalf of Escola Nacional de Saúde Pública

\section{Trigger Tool in Patient Safety: A Systematic Review of the Literature}

Keywords

Global Trigger Tool · Patient safety · Adverse events . Safety management · Patient harm · Medical errors

\section{Abstract \\ Introduction: The high incidence of adverse events (AE) is one of the troubling situations described in the litera- ture that is directly related with the rise of healthcare costs and the days of hospitalization. The Global Trigger Tool (GTT) methodology is relatively new but has shown prom- ising results, in international contexts, by tracking $\mathrm{AE}$ ac- tions. Objective: To obtain a body of proof, sets of infor- mation relating to the use of this methodology in a hos-}

\section{KARGER}

E-Mail karger@karger.com www.karger.com/pjp

Karger

Open access (c) 2017 The Author(s). Published by S. Karger AG, Basel on behalf of Escola Nacional de Saúde Pública

This article is licensed under the Creative Commons AttributionNonCommercial-NoDerivatives 4.0 International License (CC BY NC-ND) (http://www.karger.com/Services/OpenAccessLicense). Usage and distribution for commercial purposes as well as any distribution of modified material requires written permission.
Ludmila Pierdevara

Praça do Poder Local, Lote $7 \mathrm{~A}, 6^{\circ}$ frente

PR-8600-524 Lagos (Portugal)

E-Mail pierdevara@ hotmail.com 
pital setting were gathered. Methodology: This is a systematic review of the literature, based on primary studies of this subject, published between 2009 and 2014. Results: The study includes 8 primary studies and their results show relevant data on the utility of GTT in a hospital setting. Conclusion: The scientific evidence shows that the implementation of the GTT method as an AE monitoring strategy is a viable tool, since it allows to follow up the implementation of $\mathrm{AE}$ occurrence reduction changes.

(c) 2017 The Author(s). Published by S. Karger AG, Basel on behalf of Escola Nacional de Saúde Pública

\section{Introdução}

Nas últimas décadas, os avanços na tecnologia e no conhecimento criaram sistemas de saúde cada vez mais complexos. A evidência científica mostra que essa complexidade acarreta muitos riscos [1]. A elevada incidência dos eventos adversos (EA) em hospitais tem preocupado investigadores, profissionais e gestores em saúde, devido ao seu impacto socioeconómico com custos elevados para os doentes e para as instituições.

Para a Organização Mundial de Saúde, um EA é um incidente que resulta em danos para o doente. Os danos implicam prejuízo na estrutura ou funções do corpo do doente, ou qualquer efeito pernicioso daí resultante, incluindo doença, lesão, sofrimento, incapacidade ou morte, podendo ser físico, social ou psicológico [2].

Atualmente, torna-se cada vez mais imprescindível reconhecer que a identificação dos EA permite a avaliação da qualidade em saúde, contribuindo, decisivamente, para as mudanças na prática clínica. Para tal, são necessários programas e instrumentos de gestão do risco clínico, incluindo a deteção precoce e a correção dos EA.

Encontram-se descritos na literatura vários métodos de avaliação dos EA, tais como relatórios voluntários de incidentes, relatórios espontâneos com alerta, observação direta, revisão de processos clínicos, entrevista a utentes, ou a combinação destes métodos [3]. A sua seleção deve depender da sua finalidade, tendo em consideração o eventual sucesso na utilização em instituições/contextos similares [4].

Em Portugal, a Direção-Geral da Saúde, por proposta do Departamento da Qualidade na Saúde e no âmbito da qualidade organizacional, emitiu a norma 025/2012, de 19 de dezembro que orienta as instituições prestadoras de cuidados do Sistema Nacional de Saúde relativamente ao Sistema Nacional de Notificação de Incidentes e Eventos Adversos. A elaboração da presente norma baseou-se na colaboração da Agencia de Calidad Sanitaria de Andalucía. Este sistema pode ser um instrumento importante, desde que sejam, de facto, comunicados e analisados os EA. No entanto, as pesquisas mostram que a maioria dos incidentes não são comunicados pelos profissionais de saúde [5]. São conhecidos muito menos erros do que aqueles que são praticados, sendo a principal razão a culpabilização associada aos erros, o que faz com que a maioria não seja divulgada. Estima-se que os incidentes notificados voluntariamente refletem cerca de 10 a $20 \%$ dos incidentes que realmente ocorrem e destas apenas $5 \%$ causam dano ao doente [6].

Sendo assim, a resistência em assumir o erro por parte dos profissionais e em notificar voluntariamente os incidentes ocorridos dificulta aos gestores de qualidade para analisar esses erros e encontrar soluções de implementação de bons programas de gestão do risco clínico, incluindo a deteção precoce e correção de EA.

Os esforços para detetar os EA através de várias metodologias (auditorias, relatórios voluntários), são dispendiosos, com fraca sensibilidade e, na maior parte, menos eficientes [7].

$\mathrm{Na}$ tentativa de colmatar esta lacuna, o Institute for Healthcare Improvement (IHI) desenvolveu em 2002 a metodologia IHI Global Trigger Tool (GTT) para identificar os EA.

A metodologia GTT assenta numa revisão retrospetiva de processos clínicos, que utiliza pistas para identificar possíveis EA. Esta metodologia permite identificar determinadas palavras-sentinela (triggers), que poderão conduzir à identificação de um possível dano que poderá estar relacionado com um EA [8].

Para aplicar esta metodologia, o IHI aconselha que a revisão dos processos deve ser feita por um grupo de, pelo menos, dois enfermeiros, revisores primários e um médico que assume a função de revisor secundário ou árbitro. O médico não participa na revisão dos processos clínicos, mas autentica o consenso dos revisores e ajuda a classificar os resultados dos EA, em função da gravidade dos danos. Os revisores, de forma independente, duas vezes por mês, de 15 em 15 dias, selecionam, aleatoriamente, 10 dos processos que tiveram alta clínica, pelo menos há 2 meses. De forma a aumentar a validade interna da revisão, cada revisor analisa os 10 processos. A revisão é feita com base nos triggers da ferramenta GTT, e a análise de cada processo não deve exceder 20 minutos. Os processos só devem ser revistos, para identificar a presença dos triggers, e não lidos na sua totalidade. Durante a revisão, a presença de um trigger positivo encontrado, não significa, obrigatoriamente, que um EA é confirmado. No fim de cada 
mês, os revisores primários reúnem-se, com o intuito de rever todos os EA identificados e classificados em função da gravidade dos danos, de modo a chegar a um consenso. Posteriormente, realiza-se uma reunião com o médico, para autenticar o consenso dos resultados.

A ferramenta utiliza cinco categorias de danos decorrentes de um EA, sendo elas: dano temporário ao doente com intervenção necessária $(\mathrm{E})$, dano temporário que exigiu prolongamento da hospitalização $(\mathrm{F})$, dano permanente $(G)$, intervenções necessárias para sustentar a vida $(\mathrm{H})$ e a morte do doente (I). Esta classificação está de acordo com a National Coordinating Council for Medication Error Reporting and Prevention (NCC MERP). A NCC MERP classifica os erros associados ao uso de medicamentos em 9 categorias (A a I), sendo que somente as categorias E, F, G, H e I estão relacionadas com erros que ocasionam dano.

A GTT contém seis módulos que integram vários triggers, sendo eles: cuidados gerais, cirurgia, cuidados intensivos, medicação, perinatal e departamento de emergência. Quatro dos módulos são desenhados para refletir os EA que ocorrem, geralmente, em unidades especificas. Os módulos de cuidados gerais e medicação são pensados para refletir sobre os EA que podem ocorrer em qualquer unidade hospitalar.

A flexibilidade da GTT permite a sua aplicação em diversos ambientes clínicos, desde unidades ambulatórias, até unidades de cuidados intensivos. A ferramenta não é por si só uma metodologia de melhoria, mas proporciona a aquisição de dados e, consequentemente, conduz a organização à melhoria contínua [9].

A GTT é considerada uma metodologia relativamente nova, que se tem mostrado promissora em contextos internacionais.

Em virtude de se desconhecer a existência de estudos que analisem esta temática em Portugal, as implicações científicas desta revisão sistemática de literatura vão ao encontro da carência de dados científicos relacionados com a presente metodologia.

Antes de passar à metodologia da presente revisão sistemática de literatura definiu-se a questão orientadora em formato de acrónimo PICO [10] (Problema, Intervenção, Comparação e Outcomes): "Qual é a evidência científica, relativamente à utilização da metodologia GTT, no rastreio dos EA no contexto hospitalar?".

O objetivo deste trabalho consiste em obter um corpo de evidência que reúna um conjunto de informações acerca da utilização da metodologia GTT no meio hospitalar.

\section{Metodologia}

Realizou-se uma revisão sistemática da literatura, que consistiu na análise de estudos primários que se reportam à metodologia GTT. A estrutura do estudo foi baseada em princípios recomendados pelo Cochrane Handbook [11].

A priori, definiram-se as palavras-chave: Global Trigger Tool, patient safety, adverse events, safety management, patient harm e medical errors. Posteriormente, confirmou-se se as palavras-chave prévias constituíam descritores MeSH (Medical Subject Headings). Obteve-se resposta positiva para patient safety, safety management, patient harm e medical errors. Para as palavras-chave Global Trigger Tool e adverse events não se encontrou um descritor, mas por serem consideradas relevantes no estudo foram incluídas.

Seguidamente, foi realizada uma pesquisa em bases de dados online Medline via PubMed e B-on, entre julho e outubro de 2014. Como complemento, utilizou-se o motor de busca Google Scholar.

$\mathrm{Na}$ construção da estratégia de busca dos estudos, foram utilizadas as palavras-chave combinadas e/ou isoladas, e os operadores booleanos "AND"/“OR" que permitiram a ligação dos termos de pesquisa.

Foram selecionados apenas os artigos científicos cujo ano de publicação se encontra compreendido entre 2009 e 2014. De acordo com as palavras-chave utilizadas, os motores de busca identificaram 639 artigos.

A triagem dos artigos foi realizada por dois revisores de forma independente. Através de uma leitura rápida dos títulos e resumos, foram considerados, inicialmente, 24 artigos. Pela leitura do $a b$ stract, e aplicando os critérios de inclusão e exclusão, foram excluídos 15 artigos, tendo sido considerados, inicialmente, 9 estudos potencialmente elegíveis, visto terem dados suficientes para o estudo. Após a leitura na íntegra dos estudos e o consenso das duas investigadoras, foi ainda excluído um estudo, por não fornecer toda a informação considerada imprescindível à análise, perfazendo assim um total de 8 artigos selecionados.

Como critérios de inclusão, foram considerados estudos primários publicados em inglês, espanhol e português, disponíveis na íntegra, realizados com base nos processos clínicos dos doentes que estiveram internados numa unidade hospitalar e aplicaram ferramenta GTT. Foram excluídos os artigos de estudos que envolviam processos clínicos de doentes internados com idade inferior a 18 anos, ou com diagnóstico do foro psiquiátrico.

A avaliação da qualidade metodológica dos estudos incluídos foi realizada por dois investigadores independentes. Para tal, utilizou-se um teste de evidência, adaptado e utilizado por Vilelas [12]. O instrumento é composto por uma lista clara de 11 perguntas, dividido em três testes. O primeiro teste é considerado de relevância preliminar, aplicado às referências bibliográficas dos artigos, constituído por 3 questões; o segundo teste é formado por 2 questões e é aplicado aos resumos dos artigos; o terceiro é aplicado aos artigos na íntegra, sendo composto por 6 questões. Estas perguntas devem ser respondidas por cada investigador, mediante a afirmação ou a negação.

$\mathrm{Na}$ avaliação de cada artigo, foi atribuída a pontuação 1, quando o item era positivo, e zero, quando negativo. A qualidade metodológica de cada artigo foi classificada de baixa ( $0-3$ pontos), moderada (4-7 pontos), ou alta (8-11 pontos).

Para aumentar o nível de fiabilidade do estudo, realizou-se o teste de concordância entre os investigadores, através do índice de Kappa. 
Table 1. Descrição dos estudos relativos à utilização do método Global Trigger Tool (GTT)

\begin{tabular}{|c|c|c|c|}
\hline $\begin{array}{l}\text { Referência bibliográfica } \\
\text { e ano de publicação }\end{array}$ & $\begin{array}{l}\text { (a) Tipo de estudo } \\
\text { (b) Formato da publicação } \\
\text { (c) País de origem }\end{array}$ & Objetivo do estudo e período do estudo & $\begin{array}{l}\text { Classificação do } \\
\text { estudo (Vilelas } \\
[12], 2009)\end{array}$ \\
\hline $\begin{array}{l}\text { Asavaroengchai et al. } \\
{[16], 2009}\end{array}$ & $\begin{array}{l}\text { (a) Transversal retrospetivo } \\
\text { (b) Artigo } \\
\text { (c) Tailândia }\end{array}$ & $\begin{array}{l}\text { Avaliar a eficácia da ferramenta GTT na } \\
\text { identificação e classificação dos EA, em tailandeses } \\
\text { hospitalizados }\end{array}$ & 10 pontos \\
\hline Classen et al. [17], 2011 & $\begin{array}{l}\text { (a) Observacional retrospetivo } \\
\text { (b) Artigo } \\
\text { (c) EUA }\end{array}$ & $\begin{array}{l}\text { Avaliar a incidência dos EA, através de } 3 \text { métodos, } \\
\text { em } 3 \text { hospitais da Carolina do Norte: GTT, sistema } \\
\text { de notificação voluntário e triagem pela Agency for } \\
\text { Healthcare Research and Quality (AHRQ) }\end{array}$ & 9 pontos \\
\hline $\begin{array}{l}\text { Landrigan et al. [18], } \\
2010\end{array}$ & $\begin{array}{l}\text { (a)Transversal retrospetivo } \\
\text { (b) Artigo } \\
\text { (c) EUA }\end{array}$ & $\begin{array}{l}\text { Avaliar, durante } 5 \text { anos, a incidência dos EA, através } \\
\text { de GTT, em } 10 \text { hospitais da Carolina do Norte }\end{array}$ & 10 pontos \\
\hline $\begin{array}{l}\text { Rutberg et al. [19], } \\
2014\end{array}$ & $\begin{array}{l}\text { (a) Observacional retrospetivo } \\
\text { (b) Artigo } \\
\text { (c) Suécia }\end{array}$ & $\begin{array}{l}\text { Identificar e comparar a incidência dos EA, durante } \\
4 \text { anos, através de } 2 \text { métodos: GTT e sistema de } \\
\text { notificação voluntária, no Hospital Universitário de } \\
\text { Linköping }\end{array}$ & 8 pontos \\
\hline $\begin{array}{l}\text { Ganachariet al. [20], } \\
2013\end{array}$ & $\begin{array}{l}\text { (a) Observacional retrospetivo } \\
\text { (b) Artigo } \\
\text { (c) India }\end{array}$ & $\begin{array}{l}\text { Identificar os EA, através de GTT, no hospital } \\
\text { terciário de Belgaum, durante } 3 \text { meses }\end{array}$ & 10 pontos \\
\hline
\end{tabular}

De modo a determinar o nível da evidência dos estudos incluídos, foi utilizado o esquema de classificação dos níveis de evidência baseada na hierarquia, descrito por Melnyk e Fineout-Overholt [13].

Para a análise destes estudos, recorreu-se ao método quantitativo e descritivo.

Para se obter uma ideia abrangente e atual do consenso científico acerca da metodologia GTT, os dados encontrados foram estruturados numa tabela que descreve os estudos incluídos e posteriormente apresentam-se os principais resultados e conclusões encontrados em cada estudo.

\section{Resultados}

Analisando a Tabela 1, a totalidade dos estudos encontrados foram em formato de artigo. Relativamente ao tipo de estudo, 2 são transversais, 1 correlacional e 5 observacionais retrospetivos. Todos foram realizados em contex- to hospitalar e utilizaram a ferramenta GTT. No que diz respeito aos países onde os estudos foram publicados, constatou-se que não foram encontrados estudos portugueses relacionados com a presente temática.

Quanto à avaliação metodológica da qualidade dos estudos incluídos, pode-se considerar que esta revisão da literatura foi realizada com base em estudos avaliados como de alta qualidade (score 8-11).

A concordância entre os investigadores relativamente ao consenso de seleção dos artigos incluídos, através do índice de Kappa, foi de 0,992.

Seguidamente apresentam-se os principais resultados e conclusões analisados dos estudos incluídos.

No estudo de Von Plessen et al. [14] identificaram diferenças significativas entre taxas de danos encontrados em 5 hospitais, através da ferramenta GTT e taxa média dos EA foi de $25 \%$, dos quais $96 \%$ dos danos foram temporários. 
Concluíram que a GTT deve ser utilizada nos hospitais, de forma a orientar na melhoria da segurança dos doentes e sugerem a implementação noutros hospitais do país.

Rozenfeld et al. [15] observaram que cerca de 70,0\% dos processos apresentaram no mínimo um trigger, dos quais $14,4 \%$ foram considerados $\mathrm{EA}$, sendo a incidência de 26,6 por 100 doentes e por fim sugerem que a ferramenta estudada pode ser útil como técnica de monitorização e avaliação do resultado dos cuidados prestados aos doentes internados.

O estudo de Asevaroenghchai et al. [16] identificaram uma taxa média de 41 EA por 100 doentes; $53 \%$ foram de dano temporário, enquanto $51,7 \%$ eram evitáveis. Os resultados encontrados apontam que a GTT detetou mais EA do que os métodos anteriores. A maioria dos EA tiveram baixa gravidade e eram evitáveis. Sugerem a necessidade de avaliação e validação dos triggers antes de usar GTT nos serviços de internamento.

Relativamente a Classen et al. [17] encontraram 90.1\% de EA através da GTT, 1\% com sistema de notificação voluntária e $8,99 \%$ pela AHRQ. Alegaram que através dos resultados encontrados a ferramenta GTT mostrou que os EA em hospitais podem ser 10 vezes melhor identificados, comparativamente aos resultados previamente avaliados com outros métodos.

No que diz respeito ao estudo de Landrigan et al. [18], os autores identificaram 25,1 de danos por 100 admissões, sendo a taxa de incidência dos EA de $25 \%$. Afirmam que a GTT identificou taxas de danos mais elevados do que outros métodos utilizados anteriormente. Os índices de confiança e de especificidade das revisões foi elevado, contudo, houve diferenças significativas entre os resultados apresentados pelos revisores experientes e os recémformados, sugerindo a necessidade de treino da equipa revisora antes de outros estudos.

Quanto ao estudo de Rutberg et al. [19], os investigadores alegam que a GTT identificou 20,5\% de EA e com o sistema de notificação voluntário apenas $6,3 \%$ e concluem que a GTT permite identificar um número muito mais significativo de EA do que com o sistema de notificação voluntário. Estes autores sugerem que as instituições de saúde devem utilizar um conjunto de ferramentas úteis para identificar os EA, por forma a diminuir, substancialmente, os custos relacionados com a prevenção dos EA.

Ganachari et al. [20] avaliaram a taxa de EA através da ferramenta GTT. A taxa dos EA identificados foi de $18,1 \%$, estando $50,6 \%$ relacionados com a medicação. Por fim, salientam que a GTT supera os métodos tradicionais

Trigger Tool na Segurança do Doente na identificação dos EA e sugerem a aplicação da mesma em novos estudos.

Hwang et al. [21] identificaram os EA através da GTT e encontraram diferenças estatisticamente significativas entre a identificação dos triggers e a ocorrência dos EA. Dos $13,3 \%$ triggers identificados, $10 \%$ foram considerados EA. Os resultados obtidos permitiram concluir que a ferramenta GTT foi um método útil para identificar os EA num hospital coreano e a aplicação da mesma poderia ter bons resultados noutras organizações de saúde.

\section{Discussão}

Da pesquisa efetuada, verificou-se que existe uma escassez de estudos realizados em Portugal e os artigos analisados, publicados internacionalmente, são, na sua maioria, recentes (2013 e 2014).

O grau de concordância entre os investigadores acerca dos estudos incluídos, calculado através do índice de Kappa, foi considerado segundo classificação do Landis e Koch [22] perfeito.

No que diz respeito à hierarquia da evidência científica dos estudos, dado que na globalidade são de natureza descritivos, considera-se que estamos perante um nível de evidência cinco [13].

Apesar que os resultados dos estudos analisados foram diferentes, constatou-se que as conclusões dos artigos não foram discrepantes, tendo, na verdade, todos eles apresentado sugestões que apontam no mesmo sentido. Importa também salientar que a globalidade das conclusões evidencia dados relevantes acerca da utilidade da ferramenta GTT no âmbito hospitalar, para identificar os EA. Quatro estudos analisados [17-20] reforçam que a metodologia GTT supera os métodos tradicionais na identificação dos EA e salientam a importância de aplicação da mesma em outros contextos hospitalares.

Estes dados vão ao encontro do estudo realizado pelo IHI, que testaram a metodologia GTT em 86 hospitais internacionais, que participaram no desenvolvimento desta ferramenta. Com base no relatório IHI Global Trigger Tool for Measuring Adverse Events [23], verifica-se que a ferramenta GTT constitui um método fácil de usar para identificar, com precisão, os EA e medir a taxa dos mesmos ao longo do tempo. A implementação desta ferramenta não necessita de recurso a tecnologia, nem carece de recursos financeiros elevados [7].

No que diz respeito relativamente a afirmação do IHI acerca da GTT, também, estão de acordo e um grupo de investigadores portugueses, que realizaram em 2015 um 


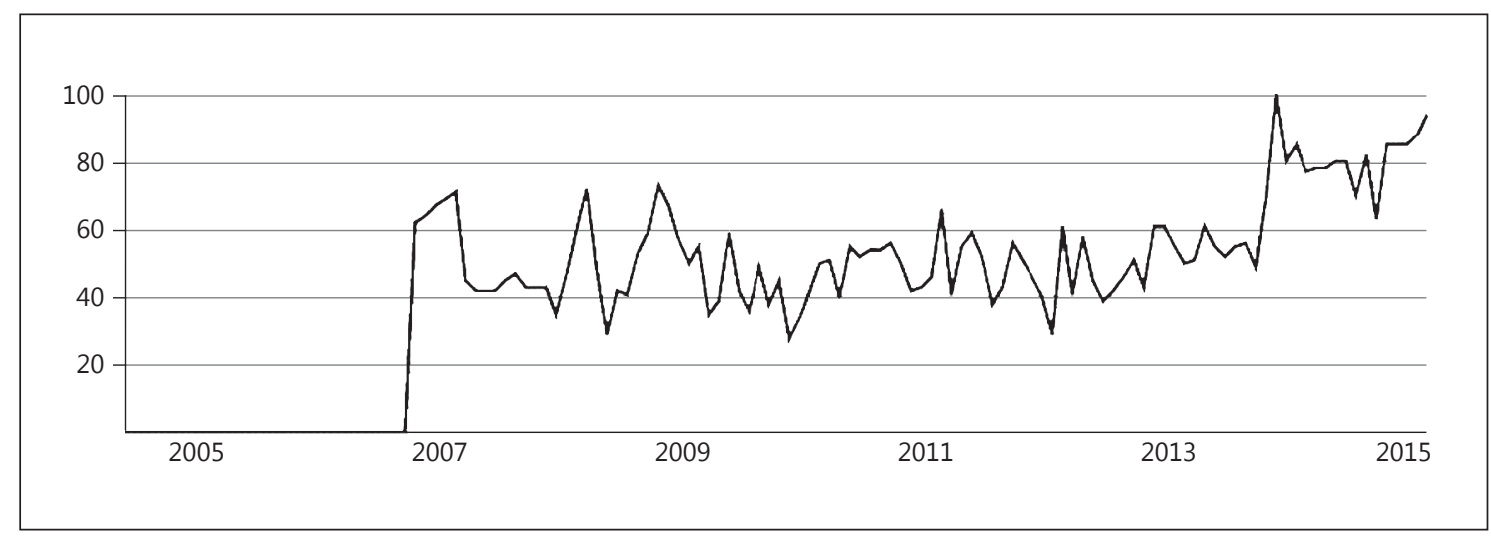

Fig. 1. A popularidade do termo Trigger Tool ao longo do tempo. Fonte: Google Trends, consultado 17/02/2015.

estudo descritivo, aplicando esta metodologia num serviço de medicina. Segundo Pierdevara et al. [24] os resultados obtidos através da aplicação da GTT refletem a real dimensão e magnitude do problema de identificar os EA ao nível institucional e sugerem que outras instituições portugueses realizem estudos desta natureza.

Esta revisão sistemática de literatura é alvo de algumas limitações. A maior limitação desta revisão foi o facto de não haver um consenso, relativamente à utilização de um instrumento padrão de avaliação das evidências da qualidade dos estudos primários.

Outra limitação deste trabalho é o reduzido número de estudos científicos relacionados com esta temática disponíveis na íntegra.

Com base nesta limitação, para explicar a escassez das publicações relacionadas com o presente tema e para assegurar que não foram excluídos estudos relevantes, pretendeu-se averiguar qual é a tendência da temática Trigger Tool a nível mundial. Sendo assim, recorreu-se à ferramenta Google Trends que permite analisar a evolução do número de pesquisas nos monitores de busca de uma palavra-chave ao longo do tempo (Fig. 1). Os resultados obtidos são fornecidos dentro de um índice entre 0 e 100 em que 100 é o ponto no tempo em que a pesquisa teve o seu máximo.

Analisando o gráfico abaixo, verificou-se que a pesquisa desta temática teve início no fim do ano 2006 e o seu máximo atingiu entre os anos 2013 e 2014. A linha de tendência sofreu uma ligeira descida em meados de 2014 e uma subida desde o fim do ano 2014 até ao presente. Portanto, examinando esta informação verificou-se que estamos perante uma área de investigação nova e em fran- co crescimento, razão pela qual não foram descartados artigos por dificuldade de não acesso.

Relativamente aos conflitos de interesse, apenas os estudos de Asavaroengchai et al. [16] e de Ganachari et al. [20] referem que não têm conflitos de interesse. Outros 6 estudos não mencionam, contudo o Classen pretense ao IHI como fundador da ferramenta.

\section{Conclusão}

A evidência científica aponta que a aplicação da metodologia GTT, como estratégia de monitorização dos EA, é uma ferramenta viável, permitindo a redução da ocorrência dos EA, fornecendo aos decisores da gestão da qualidade, informação importante para implementar medidas de melhoria contínua.

Considera-se que a temática analisada e os resultados encontrados nesta revisão de literatura são incentivadores para os profissionais de saúde ligados à prestação de cuidados criarem novos projetos no âmbito da segurança do doente, tornando-se muito útil como base metodológica para uma futura investigação nesta área. São necessários mais estudos, uma vez que não existem publicações relacionadas com a presente temática em Portugal. 


\section{Anexo I}

Grelha do teste de evidência dos artigos [12]

Teste de evidência I/teste de relevância preliminar

Questões de interesse

Sim Não

O estudo aborda o tema de interesse para a investigação?

O estudo foi publicado no período selecionado para a investigação proposta pelos investigadores?

O estudo foi publicado num idioma selecionado para a investigação?

Parecer do avaliador: Inclusão $\quad \square \quad$ Exclusão

Avaliador:

Teste de evidência II aplicado aos resumos dos artigos

Questões de interesse

Trata-se de um estudo que envolve diretamente seres humanos como sujeitos?

O estudo está direcionado para a solução do problema específico que está a ser investigado?

Parecer do avaliador: Inclusão $\quad \square \quad$ Exclusão

Avaliador:

Teste de evidência III aplicados aos artigos na integra

Questões de interesse

A definição do problema está clara?

Os objetivos do artigo estão relacionados com a questão que esta a ser alvo da revisão sistemática da literatura?

A metodologia está claramente descrita?

A investigação possui uma metodologia adequada?

A intervenção proposta pelo resultado encontrado pelo investigador é factível?

Os resultados contribuem para a prática?

Parecer do avaliador: Inclusão $\quad \square \quad$ Exclusão

Avaliador:

\section{Anexo II}

Classificação dos níveis de evidência baseada na hierarquia [13]

\begin{tabular}{|c|c|c|}
\hline Type of evidence & $\begin{array}{l}\text { Level of } \\
\text { evidence }\end{array}$ & Description \\
\hline $\begin{array}{l}\text { Systematic review } \\
\text { or meta-analysis }\end{array}$ & I & $\begin{array}{l}\text { A synthesis of evidence from all relevant } \\
\text { randomized controlled trials }\end{array}$ \\
\hline $\begin{array}{l}\text { Randomized } \\
\text { controlled trial }\end{array}$ & II & $\begin{array}{l}\text { An experiment in which subjects are randomized } \\
\text { to a treatment group or control group }\end{array}$ \\
\hline $\begin{array}{l}\text { Controlled trial } \\
\text { without } \\
\text { randomization }\end{array}$ & III & $\begin{array}{l}\text { An experiment in which subjects are nonrandomly } \\
\text { assigned to a treatment group } \\
\text { or control group }\end{array}$ \\
\hline $\begin{array}{l}\text { Case-control or } \\
\text { cohort study }\end{array}$ & IV & $\begin{array}{l}\text { Case-control study: a comparison of } \\
\text { subjects with a condition (case) with those who do } \\
\text { not have the condition (control) to determine } \\
\text { characteristics that might predict the condition } \\
\text { Cohort study: an observation of a group(s) } \\
\text { (cohort(s)) to determine the development of an } \\
\text { outcome(s) such as a disease }\end{array}$ \\
\hline $\begin{array}{l}\text { Systematic review } \\
\text { of qualitative or } \\
\text { descriptive studies }\end{array}$ & $\mathrm{V}$ & $\begin{array}{l}\text { A synthesis of evidence from qualitative or } \\
\text { descriptive studies to answer a clinical question }\end{array}$ \\
\hline $\begin{array}{l}\text { Qualitative or } \\
\text { descriptive study }\end{array}$ & VI & $\begin{array}{l}\text { Qualitative study: gathers data on human behavior } \\
\text { to understand why and how decisions are made } \\
\text { Descriptive study: provides background } \\
\text { information on the what, where, and when } \\
\text { of a topic of interest }\end{array}$ \\
\hline $\begin{array}{l}\text { Opinion or } \\
\text { consensus }\end{array}$ & VII & Authoritative opinion of expert committee \\
\hline
\end{tabular}




\section{References}

1 Balding C: From quality assurance to clinical governance. Aust Health Rev 2008;32:383391.

2 Portugal, Ministério da Saúde, Direcção-Geral da Saúde: Estrutura concetual da Classificação Internacional sobre Segurança do Doente. Lisboa, DGS, 2011.

3 Murff HJ, Patel VL, Hripcsak G, Bates DW: Detecting adverse events for patient safety research: a review of current methodologies. J Biomed Inform 2003;36:131-143.

4 Colla JB, Bracken AC, Kinney LM, Weeks WB: Measuring patient safety climate: a review of surveys. Qual Saf Health Care 2005; 14:364-366.

5 Pfeiffer Y, Manser T, Wehner T: Conceptualising barriers to incident reporting: a psychological framework. Qual Saf Health Care 2010;19:e60.

6 Sari AB, Sheldon TA, Cracknell A, Turnbull A: Sensitivity of routine system for reporting patient safety incidents in an NHS hospital: retrospective patient case note review. BMJ 2007;334:79.

7 Rozich JD, Haraden CR, Resar RK: Adverse drug event trigger tool: a practical methodology for measuring medication related harm. Qual Saf Health Care 2003;12:194-200.

8 Resar RK, Rozich JD, Classen D: Methodology and rationale for the measurement of harm with trigger tools. Qual Saf Health Care 2003; 12(suppl 2):ii39-ii45.

9 Griffin FA, Classen DC: Detection of adverse events in surgical patients using the Trigger Tool approach. Qual Saf Health Care 2008; 17: 253-258.
10 Santos C, Pimenta C, Nobre M: A estratégia PICO para a construção da pergunta de pesquisa e busca de evidências. Rev Latino Am Enferm 2007;15:2-5.

11 The Cochrane Collaboration, Higgins JP, Green S (eds): Cochrane Handbook for Systematic Reviews of Interventions. New York, John Wiley \& Sons, 2008.

12 Vilelas J: Investigação: o processo de construção do conhecimento. Lisboa, Sílabo, 2009.

13 Melnyk BM, Fineout-Overholt E: EvidenceBased Practice in Nursing \& Healthcare: A Guide to Best Practice, ed 2. Philadelphia, Wolters Kluwer Health, Lippincott Williams \& Wilkins, 2011. Available at: https://tinyurl. com/zvv77nw.

14 Von Plessen C, Kodal AM, Anhøj J: Experiences with global trigger tool reviews in five Danish hospitals: An implementation study. BMJ Open 2012;2:e001324.

15 Rozenfeld S, Giordani F, Coelho S: Eventos adversos a medicamentos em hospital terciario: estudo piloto com rastreadores. Rev Saude Publica 2013;47:1102-1111.

16 Asavaroengchai S, Sriratanaban J, Hiransuthikul N, Supachutikul A: Identifying adverse events in hospitalized patients using Global Trigger Tool in Thailand. Asian Biomed 2009;3:545-550.

17 Classen DC, Resar R, Griffin F, Federico F, Frankel T, Kimmel N, et al: "Global trigger tool" shows that adverse events in hospitals may be ten times greater than previously measured. Health Aff 2011;30:581-589.
18 Landrigan C, Parry G, Bones CB, Hackbarth AD, Goldmann DA, Sharek PJ: Temporal trends in rates of patient harm resulting from medical care. N Engl J Med 2010;363:21242134.

19 Rutberg H, Risberg MB, Sjödahl R, Nordqvist P, Valter L, et al: Characterisations of adverse events detected in a university hospital: a 4-year study using the Global Trigger Tool method. BMJ Open 2014; 4:e004879.

20 Ganachari MS, Wadhwa T, Walli S, Khoda DA, Aggarwal A: Trigger Tools for monitoring and reporting of adverse drug reactions: a scientific tool for efficient reporting. Open Access Sci Rep 2013;2:2-6.

21 Hwang J-I, Chin HJ, Chang Y-S: Characteristics associated with the occurrence of adverse events: a retrospective medical record review using the Global Trigger Tool in a fully digitalized tertiary teaching hospital in Korea. J Eval Clin Pract 2014;20:27-35.

22 Landis JR, Koch GG: The measurement of observer agreement for categorical data. Biometrics 1977;33:159-174.

23 Griffin FA, Resar RK: IHI Global Trigger Tool for Measuring Adverse Events, ed 2. Cambridge, Institute for Healthcare Improvement, 2009 (IHI Innovation Series White Paper).

24 Pierdevara L, Ventura IM, Eiras M, Gracias AM, Silva CS: Uma experiência com a Global Trigger Tool no estudo dos eventos adversos num serviço de medicina. Rev Enf Ref 2016;IV:97-105. 